\title{
EFFECT OF INTERFACE ROUGHNESS ON RESONANT TUNNELLING IN DOUBLE-BARRIER HETEROSTRUCTURES
}

\author{
T. Figielski, T. Wosiński and A. MąKosa \\ Institute of Physics, Polish Academy of Sciences \\ Al. Lotników 32/46, 02-668 Warszawa, Poland
}

\begin{abstract}
We argue that the well-boundary roughness in a double-barrier heterostructure induces subsidiary subbands in the quantum well which, in turn, lead to the appearance of a broad shoulder beyond the principal resonance peak in the current-voltage characteristics.
\end{abstract}

PACS numbers: 73.40.Gk, 85.30.Mn, 68.35.Ct

Double-barrier heterostructures (DBHs) are thought to be prospective sources of microwave and millimetre-wave power as they can exhibit a negative differential resistance (NDR) region in the current-voltage characteristics, $I(V)$. However, real DBHs very seldom display the smooth NDR region required for the controllable performance of such devices. Usually, the resonant maximum of the tunnel current versus bias voltage of DBH ends in either single-step or double-step bistability. In the latter case a broad shoulder appears between the two steps which commonly displays some structure. The view has been generally taken that the shoulder is an artifact rather than intrinsic property of $\mathrm{DBH}$ and is due to self-oscillations arising in the NDR region of the device.

This view has been called in question by Berolo et al. [1] and by Figielski et al. [2] who showed that the shoulder frequently exhibits a regular structure independent of the oscillating conditions of the device. This structure is distinctly revealed in the derivative of the current with respect to the bias voltage (Fig. 1), as well as in the photocurrent induced by a chopped illumination generating electron-hole pairs in the device $[3,4]$.

Berolo et al. [1] related the observed periodic structure to different resonance conditions in the quantum well due to a random inhomogeneity of the well thickness. It is hard, however, to explain in this way up to 12 cycles appearing within the shoulder. On the other hand, Figielski et al. [2] postulated the existence of several coupled electron-LO phonon states in the well giving rise to the satellite resonant maxima beyond the principal resonant peak. The latter presumption, in turn, has not been confirmed while broadening the statistics of measurements.

First, we argue that the shoulder is not actually due to self-oscillations in the NDR region of the principal resonance peak but results rather from the appearance 


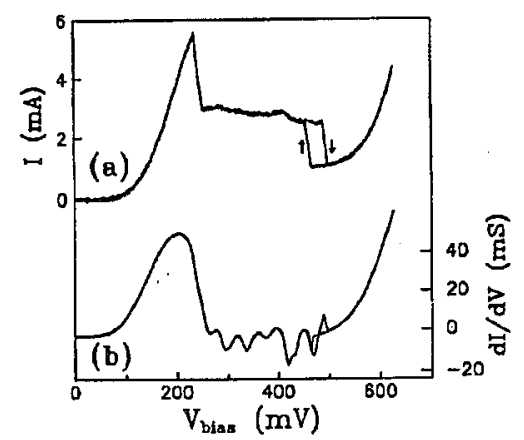

Fig. 1. Current (a) and differential conductance (b) vs. applied voltage of DBH described in Ref. [2], measured at a temperature of $80 \mathrm{~K}$ in a circuit with a $5 \Omega$ load resistor.

of subsidiary resonance maxima. The resonant $I(V)$ curve of the investigated $D B H$, shown in Fig. 1, is characteristic of a broad plateau-like shoulder. It was registered using a measuring circuit with well-defined load resistance equal to $5 \Omega$. At each bistability step the apparent $I(V)$ curve follows the load line. Thus the negative differential resistance on the right-hand side of the principal resonance peak has to be lower in the absolute value than $5 \Omega$. On the other hand, the positive differential resistance on the left-hand side of this peak, found from the $d I / d V$ curve (Fig. 1b), is about $20 \Omega$. In conclusion, the principal peak has to be asymmetric, that is tipped over the right, which is a common effect due to a charge built up in the quantum well under the resonance conditions. Hence, we can also estimate the half-width of the principal resonance peak to be of the order of several millivolts that is many times smaller than a $200 \mathrm{mV}$ wide shoulder.

After including a chip resistor (or chip capacitor) in parallel with the device, so as to minimize the inductance of the connecting leads, the self-oscillations are suppressed and the shoulder apparently disappears. This has been just the crucial argument invoked by the advocates of the self-oscillation origin of the shoulder. Instead, we have shown that after including a chip resistor the shoulder is drastically reduced but still exists, although in a residual form, as clearly seen in the $\mathrm{d} I / \mathrm{d} V$ curve in Fig. 2 . We suppose that when the relevant inductance is extremely low the total discharge of the quantum well occurs very quickly so that subsidiary resonance maxima are not explicitly revealed in the dc $I(V)$ characteristic.

In the following, we argue that interfacial roughness gives rise to the subsidiary subbands in the quantum well. The interface morphology depends on a large number of growth parameters. In general, however, the boundary roughness may be thought about as different-size islands of \pm one-monolayer height adjacent to the otherwise flat interface. Presumably, the interfacial roughness has a broad spectrum of spatial frequencies but the island sizes are generally thought to be in the micron range [5].

Electrons entering the quantum well through the emitter barrier have all in-plane components of the wave vector, $k_{\|}=\left[k_{x}, k_{y}\right]$, available in the Fermi sea 


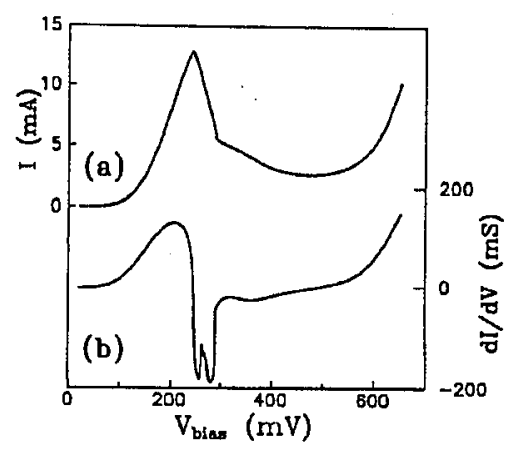

Fig. 2. Current (a) and differential conductance (b) vs. applied voltage of the same heterostructure as in Fig. 1, measured while including a $12 \Omega$ chip resistor in parallel with the device. Notice that the peak amplitude is elevated in comparison with that in Fig. 1 on the expense of the shoulder beyond the peak.

of the emitter, so as the in-plane component is conserved in tunnelling. If the well boundaries were ideally flat, an electron under resonance conditions would bounce back and forth between the two barriers while continuing its lateral motion. In reality electrons are scattered at the boundary roughness which causes their diffusive reflection and, in particular, their diffraction in the well.

In general, there may be several space frequencies in the lateral distribution of scatterers which are defined by the Fourier transform of the roughness profile. For the sake of simplicity we assume two orthogonal sets of space frequencies that amount $1 / L_{i}$ and $1 / L_{j}$ along the $x$ - and $y$-axis, respectively, where the indexes $i, j$ number particular frequencies in each set. In this case, the Laue equations of diffraction can be written as: $\Delta \boldsymbol{k}_{\|}=g_{i j}$ where $\Delta \boldsymbol{k}_{\|}$denotes the change in the wave vector caused by the scattering, and $g_{i j}=\left[2 \pi n / L_{i}, 2 \pi m / L_{j}\right]$, where $n, m$ are integers [6]. An electron, whose initial kinetic energy is sufficiently large to fulfil this condition, may be scattered into one of the subsidiary subbands induced by the well-boundary roughness. These subbands are built of the states split off from the principal subband of the quantum well and are uplifted in the energy with

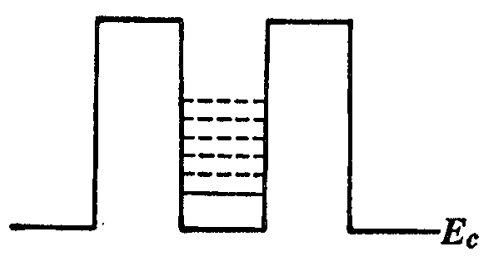

Fig. 3. The conduction-band energy diagram of DBH, where the bottoms of the principal (solid line) and subsidiary (dashed lines) subbands of the well are marked. For arbitrary parameters: $n=m=1$ and $L_{i}=L_{j}=20,27$ and $47 \mathrm{~nm}$, there are 5 subsidiary subbands with an equal spacing of $10 \mathrm{meV}$. 
respect to the latter by $E_{i j}$. The energies $E_{i j}$ are determined by the condition $2 m^{*} E_{i j} / \hbar^{2}=\left(2 \pi n / L_{i}\right)^{2}+\left(2 \pi m / L_{j}\right)^{2}$, where $m^{*}$ is the electron effective mass.

A simple example, shown in Fig. 3, illustrates the case for arbitrary parameters of the boundary roughness. It is evident that even when DBII is biased beyond the principal resonance peak, electrons can still tunnel from the emitter into the subsidiary subbands (whose number can be quite large) without violation of the in-plane-momentum conservation rule.

The presented idea has common points with the lateral spatial localization suggested by Ting et al. [7]. We have obtained some experimental results verifying this idea by examining the shoulder behaviour in a high magnetic field normal to the well boundaries.

We are greatly indebted to Dr. M. Kaniewska (Institute of Electron Technology, Warsaw) for useful discussions. This work was partly supported by the Committee for Scientific Research (Poland) under grant No. 2 P03B 03508.

\section{References}

[1] O. Berolo, E. Fortin, L. Allard, Superlattices Microstruct. 17, 163 (1995).

[2] T. Figielski, A. Mąkosa, T. Wosiński, P.C. Harness, K.E. Singer, Solid State Commun. 91, 913 (1994).

[3] P.G. Richard, E. Fortin, O. Berolo, Solid-State Electron. 33, 93 (1990).

[4] T. Figielski, A. Mąkosa, T. Wosiński, P.C. Harness, K.E. Singer, Acta Phys. Pol. A 84, 817 (1993).

[5] A. Ourmazd, in: Semiconductor Interfaces at the Sub-Nanometer Scale, Eds. H.W.M. Salemink, M.D. Pashley, Kluwer Academic Publishers, Dordrecht 1993, p. 139.

[6] C. Kittel, Introduction to Solid State Physics, 4th ed., Wiley, New York 1971.

[7] D.Z.-Y. Ting, S.K. Kirby, T.C. McGill, Appl. Phys. Lett. 64, 2004 (1994). 